\title{
Un artiste voyageur en Micronésie. L'univers flottant de Paul Jacoulet de Christian Polak et Kiyoko Sawatari (éds)
}

\section{Gilles Bounoure}

\author{
(2) OpenEdition \\ Journals \\ Édition électronique \\ URL : http://journals.openedition.org/jso/6873 \\ DOI : $10.4000 /$ jso.6873 \\ ISSN : 1760-7256 \\ Éditeur \\ Société des océanistes \\ Édition imprimée \\ Date de publication : 15 octobre 2013 \\ Pagination : 262-265 \\ ISBN : 978-2-85430-035-2 \\ ISSN : 0300-953x \\ Référence électronique \\ Gilles Bounoure, « Un artiste voyageur en Micronésie. L'univers flottant de Paul Jacoulet de Christian \\ Polak et Kiyoko Sawatari (éds) », Journal de la Société des Océanistes [En ligne], 136-137 | 2013, mis en \\ ligne le 20 octobre 2013, consulté le 23 septembre 2020. URL : http://journals.openedition.org/jso/ \\ 6873 ; DOI : https://doi.org/10.4000/jso.6873
}

Ce document a été généré automatiquement le 23 septembre 2020

(c) Tous droits réservés 


\title{
Un artiste voyageur en Micronésie. L'univers flottant de Paul Jacoulet de Christian Polak et Kiyoko Sawatari (éds)
}

\author{
Gilles Bounoure
}

\section{RÉFÉRENCE}

POLAK Christian et Kiyoko SAWATARI (éds), 2013. Un artiste voyageur en Micronésie. L'univers flottant de Paul Jacoulet, Paris, Musée du quai Branly-Somogy, 352 p., annexes, bibliogr., carte, très nombreuses ill. in et hors-texte.

1 En dépit de son étendue maritime (environ la superficie terrestre des États-Unis) et de la richesse de ses cultures traditionnelles, la Micronésie est si peu représentée dans les travaux savants et dans les collections publiques ou privées de France et de nombreux autres pays occidentaux (hors Allemagne pour des raisons bien connues) que ce volumineux catalogue d'exposition méritait d'être signalé dans ces colonnes au seul motif de ce constat. Mais cette publication, comme l'exposition qu'elle a accompagnée et qu'elle prolonge, et l'événement (faut-il dire « muséal » ou " patrimonial »?) dont elles résultent toutes deux, dépasse de beaucoup en intérêt la simple évocation d'un domaine injustement négligé. Le musée du quai Branly s'est aussi trouvé là jouer un rôle des plus légitimes, déjà exploré et exploité par maints musées allemands, justifiant son slogan de " rencontre des cultures " (largement discuté par ailleurs, voir ci-dessus sur Byrne et al., 2011) puisque certains points importants de l'histoire des arts européens, de la géopolitique du $\mathrm{xx}^{\mathrm{e}}$ siècle et de ce qu'on nommait jadis le savoir ethnographique ont ainsi été soumis au regard des visiteurs, des lecteurs et des critiques. 
2 L'exposition, présentée du 26 février au 19 mai 2013, marquait la donation au musée du quai Branly de près de trois mille pièces de la succession de Paul Jacoulet (1896-1960) restées en possession de sa fille adoptive, fort peu en comparaison des trente mille spécimens de lépidoptères qu'il avait rassemblés et dont la majorité fut vendue après sa mort au musée d'histoire naturelle d'Osaka. Si Jacoulet n'était qu'accessoirement chasseur ou collectionneur de papillons et fut surtout peintre, dessinateur et graveur, cela ne caractérise pas moins son tempérament. Né à Paris, où il ne séjourna ensuite que quelques semaines de 1907 pour y découvrir la peinture de Millet, Courbet, Gauguin, Matisse et Picasso, il quitta tout enfant la France pour le Japon où son père avait trouvé une charge d'enseignant. Il y passa lui-même le reste de sa vie, ponctuée de quelques voyages dans les zones alors sous autorité nippone comme la Micronésie, puis en diverses villes d'Occident pour y exposer ses œuvres, très directement inspirées de la technique et du style des estampes japonaises où il était passé maître et qui forment la majeure partie de cette donation.

3 Disposée sur la «mezzanine " surplombant les collections permanentes du musée, l'exposition frappait par l'abondance et l'éclat des gravures et des dessins présentés, à côté d'une sélection d'objets traditionnels malheureusement trop étriquée pour suggérer au public le raffinement extrême des cultures micronésiennes. Il était néanmoins précieux de pouvoir observer directement le travail de Jacoulet, sa maîtrise technique et ses talents de coloriste que ne restitue pas totalement le catalogue, malgré le soin remarquable dont il a bénéficié, spécialement pour la photogravure, le choix du papier et l'impression. C'est cependant à la lecture de ce volume que se révèlent certaines des questions importantes attachées à cette œuvre, et que l'exposition ne pouvait évidemment explorer.

4 Dans un musée voué aux arts graphiques (ce que n'est pas non plus la Bibliothèque nationale de France où furent présentées en 2011 pour la première fois en France soixante-dix estampes de Jacoulet), il aurait été inévitable d'évoquer, à propos d'un artiste non seulement né en France mais resté largement de culture française, le sujet $\mathrm{du}$ " japonisme » et de ses suites dans ce même pays. Les œuvres des " nabis ", de l'école de Pont-Aven, de Van Gogh et de Gauguin en sont les illustrations les plus connues. Le Français Jacoulet, devenu maître graveur spécialisé en estampes du " monde flottant » (ukiyo-e) dans la patrie même de cette imagerie, un Japon dont il semble (selon ses biographes) avoir apprécié la relative liberté sexuelle (non sans petits incidents relevés par la police, p. 44), représente certainement un exemple extrême de conversion au japonisme, aussi bien pour ses techniques que pour ses sujets et la « manière » dont il les a traités. À l'inverse, Seiki Kuroda (1866-1924), l'un des professeurs ayant enseigné l'ukiyo-é à Jacoulet, avait séjourné à Paris entre 1884 et 1893 et fut ensuite l'un des pionniers de la peinture japonaise occidentalisante (Yoga). Par ailleurs, les estampes de Jacoulet se rapprochent parfois des œuvres rapportées des mers du Sud par Max Pechstein et Emil Nolde, « images du paradis » récemment mises en valeur par plusieurs musées ethnographiques allemands.

5 Toutes les estampes ou esquisses de cette donation ne pouvaient être montrées au musée ni reproduites par le catalogue, mais il en livre une sélection très abondante, soit en pleine page soit en vignettes (pp. 244-318, un peu moins de 900, à raison de 12 par page). Une bonne partie d'entre elles concerne la Micronésie, sujet justifiant qu'il en soit question dans ces colonnes. Profitant des lignes maritimes régulières mises en place entre le Japon et les archipels saisis sur les Allemands en 1914, Jacoulet y séjourna 
à quatre reprises entre 1929 et 1932, et même tout un printemps et un été en 1930 (p. 45). Ces voyages lui inspirèrent des centaines de planches dessinées ou gravées, réalisées pour les unes assez vite, pour d'autres jusqu'à trois lustres après ses passages dans les mers du Sud. Dans leur grande majorité, elles figurent des insulaires dans leur apprêt traditionnel, mettant en valeur leurs remarquables tatouages, leur santé, leur perfection physique et la liberté de leurs mœurs amoureuses, aspects " paradisiaques » que viendront étudier Gladwin et Sarason à Chuuk en 1947, après la défaite japonaise, et dont ils tireront un classique de l'école culturaliste (1953).

Tel est l'ensemble graphique, sinon documentaire, qu'envisagent les contributions du catalogue susceptibles d'intéresser directement les océanistes. Dans « Paul Jacoulet et ses paysages rêvés des mers du Sud » (p. 19-41), Donald H. Rubinstein insiste sur le caractère idéalisé de ses figurations et la valeur symbolique de certains éléments décoratifs comme les fleurs surdimensionnées. Dans « Paul Jacoulet et la Micronésie sous mandat japonais 1929-1932» (pp. 43-47), Wakako Higuchi précise certaines circonstances des voyages de l'artiste. Au terme de "Parures et accessoires en Micronésie. Réalités et sublimation jacoulienne » (pp. 55-62), Bart Suys conclut que " son œuvre est le résultat d'observations précises, d'une remarquable érudition, d'une imagination fertile et d'un immense respect ». "Aspects ethnographiques de la Micronésie " (p. 65-73) de Manuel Rauchholz rappelle les grandes étapes de la colonisation et les principales différences culturelles entre îles hautes et basses de Micronésie. Avec "L'intime " (pp. 75-83), Beatriz Moral souligne l'écart entre la discrétion ou la clandestinité des relations amoureuses dans l'ancienne Micronésie et l'impudeur des représentations qu'en a données Jacoulet.

Dans la dernière de ces contributions, "L'art du tatouage en Micronésie ", Sébastien Galliot (pp. 85-98) rapporte les motifs dessinés ou gravés par Jacoulet à ceux qu'ont reproduits la littérature ethnographique et les relations de voyage. Il eût été utile d'y joindre d'autres sources iconographiques qu'aurait pu consulter l'artiste, notamment les gravures tirées des dessins de Louis Choris au retour de la circumnavigation de Kotzebue ou les planches 24 à 52 de l'Atlas du Voyage autour du monde de Frédéric Lütke, livrant d'après les dessins d'Alexandre Postels de bonnes observations des tatouages en usage à Pohnpei, aux Mortlock, etc. Les observations de voyageurs comme F. W. Christian (1967 [1899]), largement diffusées en leur temps, pouvaient aussi fournir à l'artiste des éléments d'information et d'interprétation, par exemple sur les tatouages des femmes à Pohnpei inspirés des tressages de leurs paniers (à côté d'autres motifs, étoiles à huit branches et cercles, p. 74), les motifs de la queue de poisson et du chevron chez les hommes à Yap (p. 268), suggérant un déclin général du tatouage dès la fin $d u x^{e}$ siècle, mais aussi la persistance de pratiques de scarifications apparemment absentes des dessins de Jacoulet mais encore relevées en 1947 par Gladwin et Sarason (1953 : 111-112) comme par LeBar (1964 : 171). En tout état de cause, S. Galliot conclut qu'une partie des dessins de

Jacoulet reproduit « les travaux de l'ethnologue japonais Kotondo Hasebe » (1918) mais que pour d'autres "ses relevés sont uniques et contribuent de manière inattendue, près de soixante-dix ans après leur production, à la connaissance iconographique du tatouage micronésien ».

8 Reste à mieux établir les conditions dans lesquelles l'artiste a pu conduire ses " observations précises » débouchant sur ces "relevés [...] uniques », pour autant qu'on adhère à ces certificats d'authenticité. Il semble assez typique de sa manière 
d'avoir figuré un jeune Chamorro de Saipan appuyé sur un grand masque tapuanu de Mortlock, archipel situé à plus de 700 kilomètres du sud des îles Mariannes, de sorte qu'on le croirait volontiers adepte de ce que l'ancien « nabi » Félix Vallotton nommait le " paysage composé ", conçu et réalisé en atelier hors de toute recherche de réalisme en fusionnant diverses " émotions " ressenties et dessinées « sur le motif » en des lieux différents. Aux îles Marshall comme dans d'autres archipels reculés, Jacoulet semble n'avoir pu faire que de "simples escales » : par exemple, arrivé le $1^{\text {er }}$ mai 1930 à Jaluit, il se trouvait dès le surlendemain à Kusiae (pp. 45-46). Surtout, où qu'il débarquât, il ne pouvait échapper à la police japonaise, qui avait autorité en tout lieu de Micronésie en ces années-là jusqu'à y représenter le gouvernement. Ce point est insuffisamment mis en lumière dans cette publication. Il n'y a que sous la plume de $S$. Galliot qu'on lit (p. 88, d'après les travaux de Dirk Spennemann consacrés aux seules îles Marshall) :

"On sait par exemple qu'en 1922 le Japon interdit la pratique du tatouage aux Marshall. "

Or c'est dans l'ensemble de la Micronésie, passée sous mandat de la Société des nations et confiée de ce fait non plus à la marine nippone mais à une administration civile mise en place en mars 1922, que le règlement de police imposé sous le nom de Nan'yo gunto keisatsu shobatsu rei fit du tatouage et de toute marque corporelle similaire un délit passible d'un maximum de trente jours de travaux forcés (Peattie, 1988: 76, 90 et surtout 327 n. 27). Le Japon n'avait pas de législation nationale en la matière, mais les gouverneurs de province avaient de longue date entrepris de réprimer cette pratique, d'abord chez les Aïnous de l'Hokkaido, puis chez les insulaires d'Okinawa à partir de 1879 (interdiction seulement levée en 1948 par les forces d'occupation américaines). Par leur sévérité et leur minutie, les dispositions policières auxquelles la Micronésie fut soumise "différaient peu de celles qu'avaient imposées aux villages nippons les shoguns de l'époque féodale " (Peattie, l. c.). Affirmer, comme le fait un autre contributeur (p. 44), que « la politique de japonisation conduite par le bureau des mers du Sud ne touche donc qu'une petite partie de la population, dont le style de vie traditionnel n'est pas encore, dans l'ensemble, mis à mal par les autorités japonaises " revient à jouer sur les mots. Certes le mépris des administrateurs nippons pour les insulaires qu'ils dénommaient les "Kanaks " les retenait de toute tentative d'assimilation, mais l'appui (y compris policier) qu'ils fournirent aux missions protestantes ou catholiques, les déportations de populations, les recrutements forcés de main-d'œuvre, dès avant le tournant de 1933 où le Japon se retira de la Société des nations et renforça le pouvoir de ses autorités navales en Micronésie, et bien d'autres traits de colonialisme avéré qu'on ne saurait énumérer ici, contredisent cette image " idéalisée » d'une politique japonaise sans conséquences pour la majorité des Micronésiens.

10 Il ne semble pas qu'on sache comment Jacoulet s'y prenait pour faire poser ses modèles - certainement loin des regards des policiers japonais ou de leurs recrues locales (voir Peattie, pp. 74-76) -, ni s'il les payait ou récompensait d'autre façon leur complaisance, aucun témoin n'ayant pu être interrogé sur ces séances remontant à plus de quatrevingts ans. Quant aux déterminations profondes de l'artiste, hormis sa fascination pour la Micronésie ou plus exactement ses aspects "paradisiaques ", quelques-unes paraissent pouvoir se déduire de ses affirmations et de ses silences. Plusieurs contributeurs le font remarquer, Jacoulet préféra toujours parler de la Micronésie " sous mandat » que de Nan'yo comme l'imposaient les autorités japonaises. Il est vrai 
qu'ayant travaillé quelque temps comme interprète pour l'ambassade de France à Tokyo (à l'époque de Paul Claudel) il avait pu y contracter le goût de la terminologie diplomatique. Mais il ne paraît pas avoir été tenté de vanter l'action des Japonais envers les "populations non encore capables de se diriger elles-mêmes » confiées à leurs soins par la Société des nations, aucune de ses planches publiées n'illustrant ce sujet. Il est même frappant qu'il se soit concentré sur une activité prohibée et une ornementation corporelle désormais à cacher sous des vêtements d'importation. Il y aurait quelques raisons de chercher dans ces figurations de jeunes gens tatoués, qu'on le voit reprendre en 1942, en pleine Guerre du Pacifique, puis en 1946 après la capitulation japonaise, une protestation sourde contre la politique impérialiste nippone, en même temps qu'un hommage discret à tout ce qu'il y avait de semiclandestin dans l'ukiyo-é, "monde flottant » des estampes hors commerce et des activités illicites, autre beau sujet d'ethnologie finissant de rendre cette exposition et ce catalogue du meilleur intérêt.

\section{BIBLIOGRAPHIE}

CHRISTIAN F. W. 1967 [1899]. The Caroline Islands. Travel in the Sea of the Little Lands, reprint, Londres, Frank Cass \& Co.

GLADWIN Thomas et Seymour B. SARASON, 1953. Truk: Man in Paradise, New York, Wenner-Gren Foundation for Anthropological Research.

LEBAR Frank M., 1964. The Material Culture of Truk, New Haven, Yale University Publications in Anthropology.

LÜTKE Frédéric, 1830. Voyage autour du Monde fait par ordre de sa majesté l'empereur Nicolas $1^{\text {er }}$ sur la corvette Le Séniavine... Atlas lithographié d'après les dessins originaux d'Alexandre Postels, Paris, Engerlmann et Compagnie.

PEATTIE Mark E., 1988. Nan'yo. The Rise and Fall of the Japanese in Micronesia, 1885-1945, Honolulu, University of Hawaii Press, Pacific Islands Monograph Series. 